\section{BIOMATERIALS}

\section{Cotton gets superpowers} Science 357, 1118-1122 (2017)

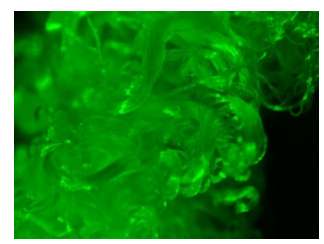

The fabrication of 'smart' textiles or wearable technologies, which respond to external stimuli, typically requires either the use of synthetic fibers or the coating or dyeing of natural fibers. These coatings or dyes are eventually worn or washed off, limiting the material's useful lifespan. Natalio et al. have now circumvented this problem by engineering cotton in which the added functionality is incorporated during fiber formation, thus preventing it from being worn away over time. After determining that the conjugation of a glucose moiety facilitated uptake of pigments into the outermost epidermal cell layer of cotton ovules cultured in vitro, the researchers synthesized two glucose-functionalized compounds for incorporation into the growing cotton fibers. Incubating fertilized ovules with one of these compounds, a glucose-conjugated 6-carboxyfluorescein (6CF-Glc), led to the production of fluorescent fibers. Substitution of 6CF-Glc with the other compound, a glucose-conjugated macrocycle caging a dysprosium(III) ion (Glc-DOTA-Dy(III)), yielded magnetic fibers instead. Various microscopic and spectroscopic methods verified that these exogenous moieties were successfully incorporated and that the morphologies of the functionalized fibers were largely consistent with that of raw fibers. In the future, similar approaches may be used for the addition of desired functionalities to other biological materials such as bamboo, silk, or flax, leading to new generations of engineered biomaterials.

$C D$

\section{HOST-MICROBE INTERACTIONS}

\section{Aiming at GPCRs Nature 549, 48-53 (2017)}

Dysbiosis of gut microbiota-the microbes residing in the gastrointestinal tract-is linked to various metabolic and neurologic conditions such as diabetes, obesity and autism. This role in regulating host physiology is likely not only through metabolism of dietary substances such as fiber, but also via secreted microbial products that regulate host signaling pathways. To identify such microbe-derived molecules, Cohen et al. performed a bioinformatics analysis of human microbiome data to find bacterial enzymes that produce $N$-acyl amides, as this class of lipids interacts with G-protein-coupled receptors (GPCRs) that have been implicated in metabolic conditions. The authors identified 143 unique $N$-acyl synthase genes, which are enriched in gastrointestinal bacteria compared to bacteria from other host sites. Individual expression of 44 of these genes in Escherichia coli indicated that they produce $\mathrm{N}$-acyl amides of six major families that are structurally similar to human GPCR ligands, including $N$-acyl serinol, which resembles the GPR119 ligand oleoylethanolamide. Profiling of one member of each of these families against 240 human GPCRs revealed strong and specific interactions, particularly among receptors expressed in the gastrointestinal tract. Mice colonized with

\title{
BACTERIAL CELL WALL
}

\section{Phages breaking free}

Nat. Microbiol. doi:10.1038/s41564-017-0023-4 (2017)

The bacterial cell wall consists of peptidoglycan (PG) and is the major obstacle in the release of newly synthesized bacteriophages. In single-strand RNA (sSRNA) phages, where replication is extremely mutagenic, diverse lysis genes have evolved at different sites within phage genomes, and their characterization could lead to new antibacterial strategies. Chamakura et al. observed that expression of the lysis gene $l y s^{M}$ from ssRNA phage $M$ causes morphological defects that are consistent with inhibition of PG biosynthesis. To identify the Escherichia coli target, the authors co-expressed $l y s^{M}$ with plasmids containing random E. coli genome fragments and isolated lysis-resistant clones containing the murJ gene. mur」 encodes a flippase that translocates the final PG precursor lipid II across the cytoplasmic membrane. The identification of Lys $^{\mathrm{M}}$-resistant MurJ mutants and in vivo flippase assays provided further evidence that Lys ${ }^{\mathrm{M}}$ specifically blocks lipid II flipping. Moreover, in vivo thiolmodification experiments in the presence and absence of Lys $^{\mathrm{M}}$ indicated that Lys ${ }^{\mathrm{M}}$ binding could inactivate MurJ by trapping it in one of its two flipping cycle conformations. Thus, Lys ${ }^{\mathrm{M}}$ adds to the growing repertoire of viral proteins that block PG biosynthesis steps and may facilitate the development of novel antibiotics.

bacteria expressing the synthase for $N$-acyl serinols showed reduced blood sugar levels in an oral glucose-tolerance test, consistent with action on host GPR119. These findings represent some of the first examples of microbe-derived small molecules affecting host physiology and highlight the use of functional metagenomics to identify important microbial effectors.

UBIQUITIN BIOLOGY

\section{Unraveling the chain}

Mol. Cell. doi:10.1016/j.molcel.2017.08.020 (2017)

Nat. Struct. Mol. Biol. doi:10.1038/nsmb.3475 (2017)

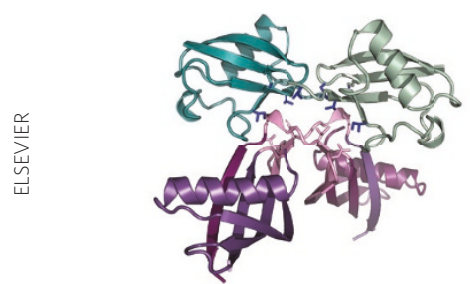

The formation of covalent chains of ubiquitin on target proteins regulates a large variety of signaling events, and different chain types, linked through ubiquitin's primary amines at M1, K6, K11, K27, K29, $\mathrm{K} 33, \mathrm{~K} 48$ and $\mathrm{K} 63$, are present in cells in varying amounts. For example, most linkages (>75\%) are formed via K48- or K63-linked chains, whereas K6-linked chains are rare $(<1 \%)$. Phage-display technology has enabled the development of antibodies for the more abundant linkage types; however, the detection of K6 chains remains challenging because of selectivity and technical issues. Michel et al. utilized phage-display-derived affimer reagents based on a non-antibody, cystatinfold protein scaffold, in which a series of randomized loops produces high-affinity binders. Structure-guided optimization of a candidate K6-specific affimer improved binding affinity, enabled detection of endogenous K6 linkages, and revealed by proteomic analysis the E3 ubiquitin ligase HUWE1 as a major source for cellular K6 chains. K6 affimers label damaged mitochondria upon activation of another E3 ligase, Parkin. Gersch et al. used affimers to reveal Parkin substrates that were modified with K6 chains upon depolarization. Together, these studies show that the development of the K6 affimer provides exciting potential for revealing new insights into ubiquitin biology. 